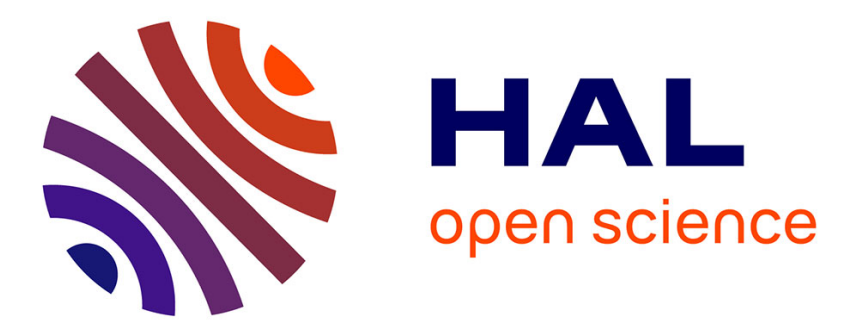

\title{
Comprehensive Evaluation of Land Resources Carrying Capacity under Different Scales Based on RAGA-PPC
}

\author{
Qiuxiang Jiang, Qiang Fu, Jun Meng, Zilong Wang, Ke Zhao
}

\section{To cite this version:}

Qiuxiang Jiang, Qiang Fu, Jun Meng, Zilong Wang, Ke Zhao. Comprehensive Evaluation of Land Resources Carrying Capacity under Different Scales Based on RAGA-PPC. 8th International Conference on Computer and Computing Technologies in Agriculture (CCTA), Sep 2014, Beijing, China. pp.200-209, 10.1007/978-3-319-19620-6_25 . hal-01420233

\section{HAL Id: hal-01420233 \\ https://hal.inria.fr/hal-01420233}

Submitted on 20 Dec 2016

HAL is a multi-disciplinary open access archive for the deposit and dissemination of scientific research documents, whether they are published or not. The documents may come from teaching and research institutions in France or abroad, or from public or private research centers.
L'archive ouverte pluridisciplinaire $\mathbf{H A L}$, est destinée au dépôt et à la diffusion de documents scientifiques de niveau recherche, publiés ou non, émanant des établissements d'enseignement et de recherche français ou étrangers, des laboratoires publics ou privés. 


\title{
Comprehensive Evaluation of Land Resources Carrying Capacity under Different Scales Based on RAGA-PPC
}

\author{
Qiuxiang Jiang ${ }^{1,2,3,4, a}$, Qiang Fu ${ }^{1,2,3}$, Jun Meng ${ }^{4}$, Zilong Wang ${ }^{1,2,3,4}$, Ke Zhao ${ }^{1}$ \\ ${ }^{1}$ College of Water Conservancy and Architecture, Northeast Agricultural University, Harbin \\ 150030; ${ }^{2}$ Collaborative Innovation Center of Grain Production Capacity Improvement in \\ Heilongjiang Province, Harbin 150030; ${ }^{3}$ Key Laboratory of Water-saving Agriculture, College \\ of Heilongjiang Province, Harbin 150030; ${ }^{4}$ Postdoctoral Mobile Research Station of \\ Agricultural and Forestry Economy Management, Northeast Agricultural University, Harbin \\ 150030 \\ ajiangqiuxiang914@163.com
}

\begin{abstract}
Land carrying capacity is an important indicator of the regional population, resources and environment evaluation of sustainable development. In this study, based on fully considering the influencing factors of land resources carrying capacity, land resources carrying capacity evaluation indicator system is constructed, composed of four sub target, i.e. the utilization of land resources, social development, economic and technology and the ecological environment. Real-coded accelerating genetic algorithm is used to optimize projection pursuit model to carry on comprehensive evaluation of land resources carrying capacity under different scales. Results show that national resources carrying capacity level of large-scale land falls under grade III under national scale; Heilongjiang Province and Sanjiang Plain belong to grade II under medium scale; The city of Jixi, Hegang, Shuangyashan and Jiamusi fall on grade I and the city of Qitai River, Muling and Yilan belong to grade II under small scale. The measures of effective utilization of land resources can be further proposed combined with the regional land resource evaluation results.
\end{abstract}

Keywords: land resources, carrying capacity evaluation, projection pursuit evaluation, Sanjiang Plain

\section{Introduction}

Land is the material basis for human survival and development, human basic necessities of life all cannot do without the land. As land resources are limited, and land resources are non-renewable, but with the rapid increase in population, socioeconomic growth, the destruction of land resources, the conflict between human demand and the limited land resources escalates and the irrational land use has threatened the sustainable development of human society and economy. Therefore, scientific evaluation of the land resources carrying capacity and rational development, utilization and protection of land, integrative distribution of land resources, make sustainable development of land resources possible. 
At present, most of scholars at home and abroad concentrate on the exploitation of land resources [1], sustainable utilization [2] optimal allocation [3], and the ecological environment evaluation [4] and so on, the research on comprehensive evaluation of land resources carrying capacity is very limited. However, comprehensive evaluation is the basis of water-related research aforementioned, Sustainable utilization of land resources can only be achieved by management within its carrying capacity. Land Resources Carrying Capacity (LRCC) is an important indicator for evaluating the regional population, resources and environment and sustainable development [5], the research has important significance on the development strategy of regional economic development, population growth, land use planning and layout of production. In view of this, land resources not only contains the natural attributes, but also contains the complex social, economic and ecological attributes. Real-coded accelerating genetic algorithm- projection pursuit model was applied to carry on comprehensive evaluation of land resources carrying capacity of Sanjiang Plain, and the comparative study was done under both national and regional scale to form scientific understanding and judgment on land carrying capacity levels. The research results can provide reference for the sustainable use of land resources and the development of society.

\section{Study Area}

Sanjiang Plain is part of the plains of the Northeast, is China's largest freshwater marsh distribution area, including Ken River, Muling River plains, Xingkai lake plains and the triangular confluence region of Heilongjiang, Songhua and Ussuri. The Sanjiang plain is an important commodity grain production base, and also the key to the realization of Heilongjiang Province billion kilogram of grain production engineering and food safety in China. Over the years, excessive pursuit of economic benefits results in the increasing of plant area in Sanjiang plain [6], single plant structure. Coupled with the absence of appropriate government, Soil quality degraded severely, resulting in the increase of the proportion of low yielding fields. The utilization of land resources in Sanjiang Plain is regarded to be unreasonable, a series of problems has appeared, such as the low utilization efficiency and the decreasing of the carrying capacity, which has imposed a serious threat to the sustainable use of resources in Sanjiang Plain and sustainable socio-economic development. Therefore, in the premise of ensuring national food security and regional ecological security, scientific evaluation of land resources carrying capacity, the rational allocation of land resources, improving the land resource sustainable support ability in industrial and agricultural production and the development of social economy, seems to be basis of the realization of Sanjiang plain soil resources sustainable utilization.

\section{Research Methods}

This study will combine real coding based Accelerating Genetic Algorithm (RAGA) and Projection Pursuit Classification Model (PPC) organically. RAGA is used to 
optimize the parameters of projection direction in PPC, conversing the highdimensional data to a low dimensional space, that is to say, multiple evaluation indicators are synthesized into an integrated one, then sort by projection value and recognition [7].

\subsection{PPC Model}

PPC model modeling steps are as follows [8-10]:

Step 1: Indicators normalized. The evaluation sample set is $\left\{x^{*}(i, j) \mid i=1,2, \ldots, n, j=1,2, \ldots, p\right\}$, where $x^{*}(i, j)$ is the $j$ th indicator value of $i$ th sample, $n$ is the number of the samples $p$ is the number of the indicators. In order to eliminate the variation range of each indicator value dimension and unified the indicator value, the type of normalization, using the following formula for normalized.

The bigger the better indicators (forward): $x(i, j)=\frac{x^{*}(i, j)}{x_{\max }(j)}$

The smaller the better indicators (reverse): $x(i, j)=\frac{x^{*}(i, j)}{x_{\max }(j)}$

Where $x_{\max }(j) 、 x_{\min }(j)$ are the biggest and smallest value of the $j$ th indicator; And $x(i, j)$ is the normalized sequence.

Step 2: Constructing projection indicator function $Q(a)$. Projection pursuit method is process of projecting the $\mathrm{P}$ dimension data $\{x(i, j) \mid j=1,2, \ldots, p\}$ into one dimension projection value $z(i)$ in the direction of $a=\{a(1), a(2), a(3), \ldots, a(p)\}$

$$
z(i)=\sum_{j=1}^{p} a(j) x(i, j) \quad(i=1,2, \ldots, n)
$$

Where $a$ is the unit vector. Then do the classification according to the one dimensional scatter diagram of $\{z(i) \mid i=1,2, \ldots, n\}$. When projecting the indicators, the scattered feature of indicator $z(i)$ should be as densely as possible for region projection point while as dispersed as possible for the whole projection point. Therefore, the projection indicator function can be expressed as:

$$
Q(a)=S_{z} D_{z}
$$

Where $S_{z}$ is the standard deviation of indicator $z(i), D_{z}$ is the regional $\mathrm{d}$ ensity of $z(i)$, that is

$$
\begin{gathered}
S_{z}=\sqrt{\frac{\sum_{i=1}^{n}(z(i)-E(z))^{2}}{n-1}} \\
D_{z}=\sum_{i=1}^{n} \sum_{j=1}^{n}(R-r(i, j)) \cdot u(R-r(i, j))
\end{gathered}
$$

Where $E(z)$ is the average value of $\{z(i) \mid i=1,2, \ldots, n\}, \mathrm{R}$ is the window radius of the regional density, the usual value is $0.1 S_{z} ; r(i, j)$ is the distance between samples, $r(i, j)=|z(i)-z(j)| ; \quad u(t)$ is the unit step function, when $t \geq 0$, it is 1 , when $t<0$, it 
is 0 .

Step 3: Optimizing the projection indicator function. When the sample set of indicators is given, the projection indicator function only varies with projection direction $a$. The direction of projection reflects the different characteristics of different data structure, the best projection direction projection direction can maximize the feature structure of high dimensional data, so we can estimate the best projection direction through solving the maximum of the projection indicator function, that is:

$$
\begin{aligned}
& \text { Objective function: } \quad \max : Q(a)=S_{z} \cdot D_{z} \\
& \text { Constraints: } \quad \text { s.t. }: \sum_{j=1}^{p} a^{2}(j)=1
\end{aligned}
$$

This is a complex nonlinear optimization problem, in which $\{a(j) \mid j=1,2, \ldots, p\}$ is the decision variables. Traditional optimization method is difficult to handle this problem, so in this paper, accelerated real-coded genetic algorithm is used to address the high dimensional global optimization problems.

Step 4: Comprehensive Evaluation. The best projection direction $a^{*}$ obtained by 3 was put into formula (3) to acquire projection value $z^{*}(i)$ of each sample, i.e. the comprehensive evaluation value of land resources carrying capacity.

\subsection{Accelerated Real-coded Genetic Algorithm}

The genetic algorithm, put forward by Professor Holland of the University of Michigan's America, is a kind of adaptive global optimization probability search algorithm simulating heredity and evolution of biology in the natural environment. It mainly includes selection, crossover and mutation operations. The specific steps are as follows $[7,11]$ :

Step 1: randomly generate $\mathrm{N}$ groups' random variables.

Step 2: calculate the objective function value, sorted by size, from largest to smallest.

Step 3: calculate the evaluation function based on ranking.

Step 4: selection operation, generating new population.

Step 5: crossover the new population generated in step 4.

Step 6: mutate the new population generated in step 5 .

Step 7: evolutionary iteration.

Step 8: take the variable interval of outstanding individual generated in step 1 and 2 as the variable initial change interval, then run step 1 step 7, bringing about accelerated process, until the function value of best individual less than the default value or run number reaches the predetermined value. At this time, the best individual in the current population is treated as RAGA results. 


\section{Comprehensive evaluation of land resource carrying capacity}

\subsection{Construction of Evaluation Indicator System}

Land resources carrying capacity evaluation indicator system (Fig.1) was constructed according to the principle of constructing evaluation indicator system, comprehensive analysis on the regional land resources and influence factors, the experience of other scholars' research achievements [12-14] and the status of the ecological environment. Evaluation system is divided into the total objective layer, sub objective layer and indicator layer, where the indicator layer contains 29 indicators.

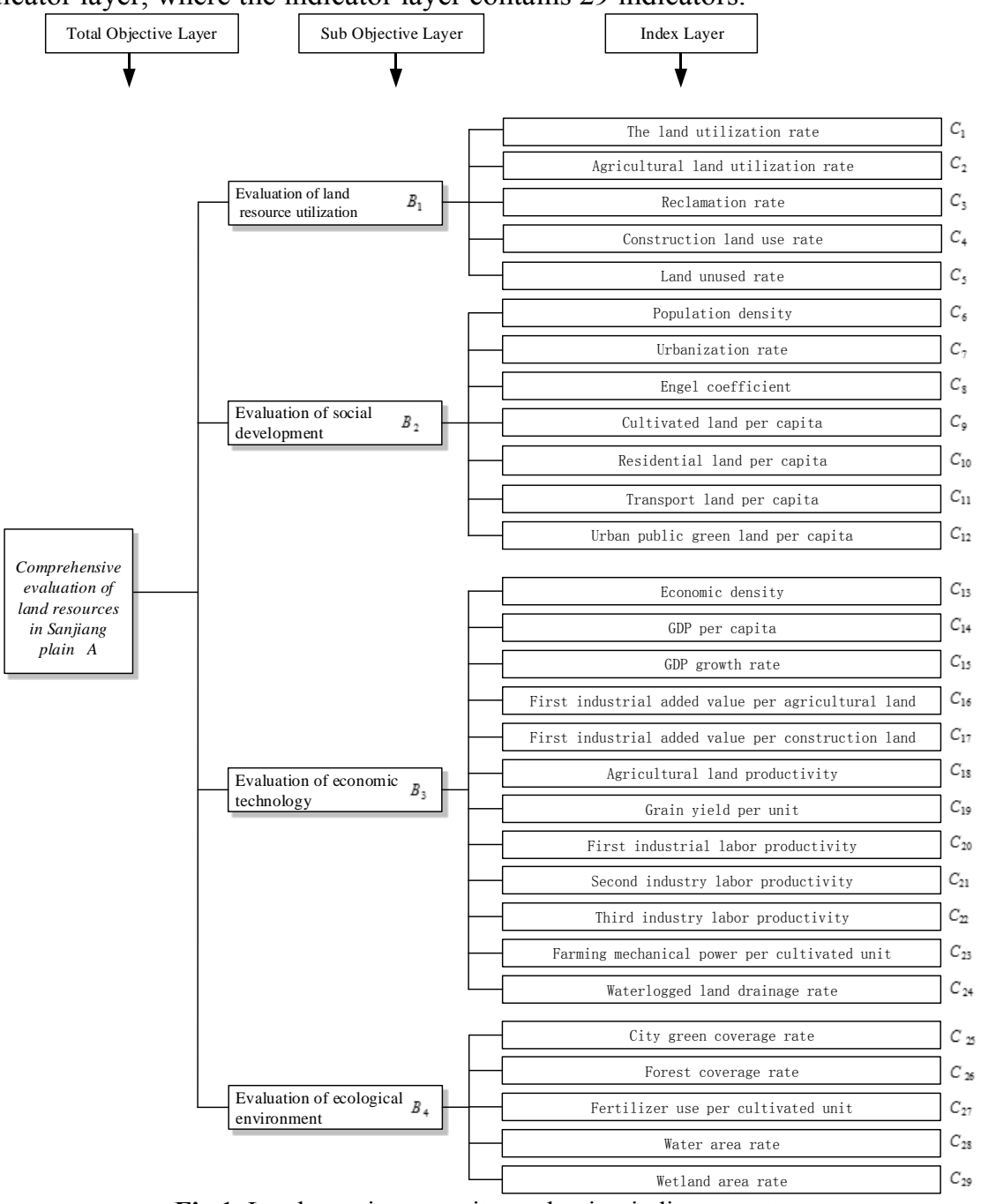

Fig.1. Land carrying capacity evaluation indicator system 


\subsection{Comprehensive Evaluation of Land Resources Carrying Capacity}

In order to be more scientific and reasonable on the evaluation of the land resources carrying capacity in Sanjiang plain, this study selected 10 land samples for carrying capacity evaluation, including the national large-scale, the Heilongjiang Province and Sanjiang plain under medium scale and seven cities in Sanjiang plain under small scale. Value table of land resources in different scale statistics 2012 carrying capacity evaluation indicator. The comprehensive evaluation indicator value of land resources carrying different scale in 2012 is shown in Table 1.

Matlab is used to program RAGA-PPC model to evaluate the total target and four sub goals of land resource carrying capacity under different scale. The total goal, the best projection value (i.e. $Z^{*}$ ) of sub goal and its ranking are shown in Table 2.

On the basis of accumulation and dispersion characteristics of land resource carrying capacity value, coupled with the comprehensive evaluation of the land resources carrying capacity in Heilongjiang Province, land resources carrying capacity evaluation level can be divided into three grade: grade I $Z_{A}^{*} \geq 3.30$, land resources carrying capacity is strong, at stress-free state; grade II ${ }^{2.6}<Z_{A}^{*}<3.30$, land resources carrying capacity belongs to the medium level, at stable state; grade III $Z_{A}^{*} \leq 2.6$, land resources carrying capacity is weak, in a state of stress. Comprehensive evaluation result of land resource carrying capacity under different scale in 2012 is shown in Table 2.

Table 2. Comprehensive evaluation result of land resource carrying capacity under different scale in 2012

\begin{tabular}{|c|c|c|c|c|c|c|c|c|c|c|}
\hline \multirow{2}{*}{$\begin{array}{c}\text { Evaluation } \\
\text { Objection }\end{array}$} & \multicolumn{7}{|c|}{ Small scale } & \multicolumn{2}{|c|}{ Medium scale } & \multirow{2}{*}{$\begin{array}{l}\begin{array}{c}\text { Large } \\
\text { scale }\end{array} \\
\text { China }\end{array}$} \\
\hline & Jixi & $\begin{array}{c}\text { Hegan } \\
\mathrm{g}\end{array}$ & $\begin{array}{c}\text { Shuangyas } \\
\text { han }\end{array}$ & Jiamus & Qitahe & Muling & Yilan & $\begin{array}{c}\text { Sanjiang } \\
\text { Plain } \\
\end{array}$ & $\begin{array}{c}\text { Heilongjiang } \\
\text { province }\end{array}$ & \\
\hline \multirow{2}{*}{$\begin{array}{cc}Z_{1}^{*} \\
B_{1} \begin{array}{c}\text { Ranking } \\
\end{array}\end{array}$} & 1.32 & 1.12 & 1.66 & 1.63 & 1.56 & 1.18 & 1.48 & 1.40 & 1.16 & 0.74 \\
\hline & 6 & 9 & 1 & 2 & 3 & 7 & 4 & 5 & 8 & 10 \\
\hline \multirow{2}{*}{$\begin{array}{cc}B_{2}^{*} \\
\text { Ranking }\end{array}$} & 1.43 & 1.50 & 1.71 & 1.87 & 0.95 & 1.54 & 1.49 & 1.55 & 1.22 & 0.70 \\
\hline & 7 & 5 & 2 & 1 & 9 & 4 & 6 & 3 & 8 & 10 \\
\hline \multirow{2}{*}{ 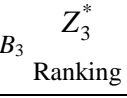 } & 1.18 & 1.02 & 1.11 & 1.12 & 1.30 & 1.36 & 1.44 & 1.16 & 1.61 & 2.51 \\
\hline & 6 & 10 & 9 & 8 & 5 & 4 & 3 & 7 & 2 & 1 \\
\hline \multirow{2}{*}{$\begin{array}{cc}B_{4} & Z_{4}^{*} \\
& \text { Ranking }\end{array}$} & 1.66 & 0.98 & 0.90 & 1.28 & 0.60 & 0.37 & 0.62 & 1.09 & 0.59 & 0.32 \\
\hline & 1 & 4 & 5 & 2 & 7 & 9 & 6 & 3 & 8 & 10 \\
\hline \multirow{2}{*}{ A $\begin{array}{c}Z_{A}^{*} \\
\text { Ranking }\end{array}$} & 3.47 & 3.34 & 3.49 & 3.35 & 2.76 & 2.77 & 3.11 & 3.23 & 2.67 & 2.12 \\
\hline & 2 & 4 & 1 & 3 & 8 & 7 & 6 & 5 & 9 & 10 \\
\hline $\begin{array}{c}\text { Evaluation } \\
\text { grade }\end{array}$ & I & I & I & I & II & II & II & II & II & III \\
\hline
\end{tabular}


Table 1. The comprehensive evaluation indicator value of land resources carrying different scale in 2012

\begin{tabular}{|c|c|c|c|c|c|c|c|c|c|c|c|c|}
\hline \multirow{2}{*}{ Indicator } & \multirow{2}{*}{ Unit } & \multirow{2}{*}{ Type } & \multicolumn{7}{|c|}{ Small scale } & \multicolumn{2}{|c|}{ Medium scale } & \multirow{2}{*}{$\begin{array}{c}\text { Large scale } \\
\text { China }\end{array}$} \\
\hline & & & Jixi & Hegang & Shuang-yashan & Jiamusi & Qitahe & Muling & Yilan & Sanjiang Plain & Heilongjiang province & \\
\hline $\mathrm{C}_{1}$ & $\%$ & $\mathrm{~F}$ & 84.28 & 90.78 & 92.86 & 87.22 & 96.97 & 98.44 & 95.41 & 89.81 & 87.1 & 72.21 \\
\hline $\mathrm{C}_{2}$ & $\%$ & $\mathrm{~F}$ & 79.8 & 86.52 & 88.59 & 82.78 & 91.41 & 90.06 & 90.53 & 85.11 & 83.8 & 68.43 \\
\hline $\mathrm{C}_{3}$ & $\%$ & $\mathrm{~F}$ & 43.74 & 37.61 & 48.05 & 62.91 & 45.26 & 23.82 & 52.89 & 48.74 & 26.16 & 12.68 \\
\hline $\mathrm{C}_{4}$ & $\%$ & $\mathrm{~F}$ & 4.36 & 4.09 & 4.16 & 4.29 & 5.53 & 2.01 & 4.65 & 4.2 & 3.3 & 3.44 \\
\hline $\mathrm{C}_{5}$ & $\%$ & $\mathrm{R}$ & 0.13 & 0.33 & 0.06 & 0.1 & 0.09 & 0.07 & 0.14 & 0.13 & 0.09 & 0.24 \\
\hline $\mathrm{C}_{6}$ & people $/ \mathrm{km}^{2}$ & $\mathrm{R}$ & 83 & 74 & 68 & 74 & 149 & 44 & 88 & 78 & 85 & 141 \\
\hline $\mathrm{C}_{7}$ & $\%$ & $\mathrm{~F}$ & 63.91 & 80.74 & 64.3 & 50.08 & 58.87 & 49.48 & 42.26 & 60.12 & 56.9 & 52.57 \\
\hline $\mathrm{C}_{8}$ & $\%$ & $\mathrm{R}$ & 33.82 & 34 & 29.8 & 35.1 & 38.21 & 39.61 & 35.2 & 33.35 & 36.9 & 36.2 \\
\hline $\mathrm{C}_{9}$ & $\mathrm{hm}^{2} /$ capita & $\mathrm{F}$ & 0.53 & 0.51 & 0.7 & 0.85 & 0.3 & 0.55 & 0.6 & 0.63 & 0.31 & 0.09 \\
\hline $\mathrm{C}_{10}$ & $\mathrm{~m}^{2} /$ capita & $\mathrm{F}$ & 303 & 294 & 311 & 287 & 222 & 270 & 286 & 288 & 255 & 156 \\
\hline $\mathrm{C}_{11}$ & $\mathrm{~m}^{2} /$ capita & $\mathrm{F}$ & 173 & 206 & 216 & 287 & 110 & 168 & 197 & 196 & 195 & 118 \\
\hline $\mathrm{C}_{12}$ & $\mathrm{~m}^{2} /$ capita & $\mathrm{F}$ & 15.4 & 26 & 15.3 & 16.2 & 26.1 & 8.2 & 28.2 & 18.5 & 19.3 & 12.3 \\
\hline $\mathrm{C}_{13}$ & Yuan $/ \mathrm{km}^{2}$ & $\mathrm{~F}$ & 259 & 244 & 256 & 206 & 483 & 221 & 260 & 251 & 303 & 541 \\
\hline $\mathrm{C}_{14}$ & $10^{4}$ Yuan/ capita & $\mathrm{F}$ & 3.1 & 3.3 & 3.7 & 2.8 & 3.2 & 5.1 & 3 & 3.5 & 3.6 & 3.8 \\
\hline $\mathrm{C}_{15}$ & $\%$ & $\mathrm{~F}$ & 13.6 & 13.5 & 13.5 & 13.8 & 8.3 & 17 & 12.3 & 13.2 & 10.1 & 9.7 \\
\hline $\mathrm{C}_{16}$ & $10^{4}$ Yuan $/ \mathrm{km}^{2}$ & $\mathrm{~F}$ & 92.03 & 83.15 & 94.65 & 75.04 & 54.08 & 43.43 & 84.95 & 80.68 & 55.74 & 79.73 \\
\hline $\mathrm{C}_{17}$ & $10^{4}$ Yuan $/ \mathrm{km}^{2}$ & $\mathrm{~F}$ & 4249 & 4217 & 4148 & 3348 & 7840 & 9040 & 3937 & 4344 & 7760 & 14108 \\
\hline $\mathrm{C}_{18}$ & $10^{4}$ Yuan $/ \mathrm{hm}^{2}$ & $\mathrm{~F}$ & 1.11 & 0.59 & 0.81 & 1.14 & 0.84 & 2.12 & 1.8 & 1.06 & 1.96 & 3.86 \\
\hline $\mathrm{C}_{19}$ & $\mathrm{~kg} / \mathrm{hm}^{2}$ & $\mathrm{~F}$ & 3305 & 2061 & 2648 & 4123 & 3131 & 2679 & 7445 & 3522 & 4867 & 4843 \\
\hline $\mathrm{C}_{20}$ & Yuan/ capita & $\mathrm{F}$ & 5.88 & 10.42 & 8.56 & 3.58 & 2.77 & 4.53 & 5.68 & 5.39 & 2.65 & 2.03 \\
\hline $\mathrm{C}_{21}$ & Yuan/ capita & $\mathrm{F}$ & 12.12 & 11.28 & 18.39 & 11.55 & 11.65 & 26.86 & 8.24 & 13.16 & 16.13 & 10.12 \\
\hline $\mathrm{C}_{22}$ & Yuan/ capita & $\mathrm{F}$ & 5.88 & 4.58 & 5.18 & 6.68 & 5.02 & 4.29 & 5.47 & 5.63 & 7.3 & 8.36 \\
\hline $\mathrm{C}_{23}$ & $\mathrm{kw} / \mathrm{hm}^{2}$ & $\mathrm{~F}$ & 1.93 & 1.49 & 1.47 & 1.65 & 2 & 1.48 & 2.53 & 1.7 & 3.85 & 8.43 \\
\hline $\mathrm{C}_{24}$ & $\%$ & $\mathrm{~F}$ & 56.9 & 82.2 & 73.9 & 50.3 & 61.8 & 60.4 & 80.2 & 65.8 & 75.3 & 51 \\
\hline $\mathrm{C}_{25}$ & $\%$ & $\mathrm{~F}$ & 12.77 & 19.26 & 10.44 & 11.95 & 39.01 & 3.57 & 11.69 & 13.81 & 16.31 & 24.67 \\
\hline $\mathrm{C}_{26}$ & $\%$ & $\mathrm{~F}$ & 32.6 & 45.9 & 37.7 & 16.6 & 43.8 & 65.7 & 35.1 & 33.4 & 42.6 & 20.4 \\
\hline $\mathrm{C}_{27}$ & $\mathrm{~kg} / \mathrm{hm}^{2}$ & $\mathrm{R}$ & 46.6 & 76.1 & 58.7 & 99.2 & 48.8 & 58.3 & 90.8 & 74.8 & 203 & 479.7 \\
\hline $\mathrm{C}_{28}$ & $\%$ & $\mathrm{~F}$ & 11.69 & 6.8 & 5.97 & 10.02 & 2.55 & 0.88 & 4.41 & 7.89 & 1.91 & 1.72 \\
\hline $\mathrm{C}_{29}$ & $\%$ & $\mathrm{~F}$ & 15.59 & 8.89 & 7.07 & 12.69 & 2.94 & 1.48 & 4.45 & 10.05 & 9.53 & 4.01 \\
\hline
\end{tabular}

Note: 1, the data on cities (counties) in Sanjiang plain and Heilongjiang province evaluation indicators are in reference [15]; 2, national evaluation indicator data are in reference [16]; 3, F stands for forward, $R$ stands for reverse. 


\subsection{Land Resources Carrying Capacity Evaluation and Regional Differences}

Land resources carrying capacity evaluation on national large-scale shows that in 2012 the national land resource carrying capacity is much lower than that in Heilongiiang province and the Sanjiang plain, the land resources utilization degree is low; Because of population pressure, land resources support capacity for social development and ecological environment protection is low, in the lower level of land resources carrying capacity (grade III), at a high stress level. But the national economic and technological level is high (economic and technical evaluation ranks 1 st), hence, we can control the population while increasing funding for land resources for land resources and technology development and management improvement, hence enhance the overall land resources carrying capacity, reducing the social development pressure on land resources.

Land resources carrying capacity evaluation on medium scale shows that because of the rich land resources in Heilongjiang province and Sanjiang plain and the low population density, the evaluation of land resource utilization, the supporting capacity of land resources for social development and ecological environment protection, the comprehensive land resources carrying capacity are higher than the national level, the land resources carrying capacity belongs to middle grade (III) and the use of land resources and social development are at the equilibrium state. Because of the characteristics of geographic location and natural resources, the production activity of Heilongiiang province and Sanjiang plain dominated by agricultural production, industrial structure is irrational, backward in economy. Therefore, in the economic and technical evaluation, the economic and technical level of Heilongjiang province and Sanjiang Plain are lower than the national average level, restricting the advantage role of land resources in this area.

Especially as the commodity grain production base, the Sanjiang plain should strengthen transformation of low yielding fields, put the cultivation techniques of high yield and high yield varieties into practice to comprehensively promote the agricultural production level, improve agricultural land output efficiency, grain production capacity, at the same time, efforts should be made to expand the area of basic farmland protection, improve the role and status of agricultural product base, the formation of high yielding agriculture industry clusters and the industrial chain as soon as possible, hence ensure the use and coordination of economic and technical development of land resources.

Because of the different distribution of natural resource, land resources carrying capacity in Sanjiang plain under small scale varies significantly. The land resources carrying capacity of Jixi City, Hegang City, Shuangyashan city and Jiamusi city are at a high level (grade I), in a stress-free state. The area is rich in land resources, the available land resources quantity is enough to ensure the social and economic development. Future utilization of land resources should be focused on the depth development resources, the rational allocation of land resources, at the same time it should combine with the characteristics of regional resources to adjust the industrial structure, improve the efficiency of resources, to ensure the benign circulation of land resources. 
The land resources carrying capacity of Qitaihe City, Muling City and Yilan County in Sanjiang plain belongs to the medium level (grade II), the use of the land resource and social economic development scale is in equilibrium. The area is the area of Sanjiang plain in the highest population density in the area, with the expansion of social development scale, shortage of land resources will become a limiting factor to the social and economic development. So in the future, population density should be controlled while promoting the rational distribution of industrial land, strengthening mining land reclamation, increasing the utilization of barren hills and wasteland, expanding the available land area to reduce the pressure on land resources. The efforts should also be put on the environment protection, the rational development of forest and wetlands, and all the exploitation should be on the premise of ecological environment.

\section{Conclusions}

(1) In the full analysis of land resources carrying capacity and regional characteristics, established the regional land resource carrying capacity evaluation indicator system, we set up the regional land resource carrying capacity evaluation indicator system. This system contains one total goal, four sub goals and 29 indicators, evaluating the regional land resource carrying capacity from four perspectives (i.e. land resources utilization, social development, and economy, technological and ecological environment).

(2) The paper constructed comprehensive evaluation model of land resources carrying capacity and acquired the comprehensive evaluation value of land resources carrying capacity under large scale (nation), medium scale (Heilongjiang province, Sanjiang plain), small scale (6 cites and 1 county in Sanjiang plain) in 2012.

(3) After full consideration of the comprehensive evaluation value of land resources carrying capacity, we put land resources carrying capacity into three levels. Among them, the national land resources carrying capacity falls on grade III; Heilongjiang province and Sanjiang plain under medium scale belongs to grade II; Jixi city, Hegang city, Shuangyashan city and Jiamusi city under small scale belong to grade I, while Qitahe city, Muling city and Yilan city belongs to fall on grade II. The result concluded in the paper, coupled with consideration of the characteristics of the regional social and economic development could support the policy making.

\section{Acknowledgment}

The study was supported by the National Natural Science Foundation of China (51179032, 51279031, 51209038), Cultivation Plan of Excellent Talents in New Century of Ministry of Education of Heilongjiang (1155-NCET-004), Ministry of Water Resources' Special Funds for Scientific Research on Public Causes (201301096-0204), Scientific Research Fund of Heilongjiang Provincial Education Department (12531009), Doctor Research Fund of Northeast 
Agricultural University (2012RCB58) and Heilongjiang Postdoctoral Fund (LBHZ13049).

\section{References}

1. Liu Yansui. Optimal allocation of regional land use [M]. Beijing: Academic Press, 1999.

2. Hu Yanlan, Yang Zhifeng. Method for ecological benefit assessment of rural land consolidation [J]. The Chinese society of Agricultural Engineering, 2004, 20(5): 275-280.

3. Wang Xiulan, Bao Yuhai. Study on the methods of land use dynamic change research [J]. Progress in geography, 1999, 18(1): 81-87.

4. Food and Agriculture Organization of the United Nations, FESLM: An International Framework for Evaluating Sustainable Land Management [R].World Soil Resources Report 73, 1993.

5. Slesser M, Hounam I. Carrying Capacity Assessment [R]. Report to UNESCO and FAO, Rome, 1984.

6. Jiang Qiuxiang, Fu Qiang, Wang Zilong, Jiang Ning. Spatial matching patterns of land and water resources in Sanjiang plain [J]. Journal of natural resources, 2011, 26(2): 270-277.

7. Fu Qiang, Zhao Xiaoyong. Principle and application of projection pursuit model [M]. Beijing: Science Press, 2006.

8. Fu Qiang, Wang Zhiliang, Lian Chuan. Applying PPC model based on RAGA in evaluating soil quantity variation [J]. Journal of soil and water conservation, 2002, 16(5): 109-110.

9. Xiang Jingtian, Shi Jiuen. The statistical method of data processing in nonlinear system [M]. Beijing: Science Press, 2000.

10. Zhang Xinli. Projection pursuit and its application in hydrology and water resources [D]. Chengdu: Sichuan University, 2000.

11. Zhou Ming, Sun Shudong. The principle of genetic algorithm and its application [M]. Beijing: National Defense Industry Press, 2000

12. Wang Shuhua, Mao Hanying. Design and evaluation on the indicator system of land comprehensive carrying capacity [J]. Journal of natural resources, 2001, 16(3): 248-254.

13. Zhu Ying. Study on the integrated assessment system of land resources carrying capacity for Chongming Island [D]. East China Normal University, 2007.

14. Ying Jie, Li Jingsheng. Evaluation of land resources carrying capacity in the process of urbanization. The construction of small towns, 2010 (5): 31-37.

15. Heilongiang Provincial Bureau of Statistics. Statistical yearbook of Heilongjiang Province [Z]. Beijing: Chinese Statistics Press, 2013.

16. The people's Republic of China National Bureau of Statistics. China statistical yearbook [Z]. Beijing: China Statistics Press, 2013. 